DOI: 10.15593/perm.mech/2014.3.02

УДК 532.517.2:539.3

\author{
Р.В. Агеев ${ }^{1}$, Е.Л. Кузнецова ${ }^{2}$, Н.И. Куликов ${ }^{2}$, \\ л.И. Могилевич ${ }^{1}$, В.С. Попов ${ }^{3}$ \\ ${ }^{1}$ Поволжский филиал Московского государственного университета \\ путей сообщения, Саратов, Россия \\ ${ }^{2}$ Московский авиационный институт (национальный \\ исследовательский университет), Москва, Россия \\ ${ }^{3}$ Саратовский государственный технический университет \\ имени Ю.А. Гагарина, Саратов, Россия \\ МАТЕМАТИЧЕСКАЯ МОДЕЛЬ ДВИЖЕНИЯ \\ ПУЛЬСИРУЮЩЕГО СЛОЯ ВЯЗКОЙ ЖИДКОСТИ \\ В КАНАЛЕ С УПРУГОЙ СТЕНКОЙ
}

\begin{abstract}
Поставлена и аналитически решена задача гидроупругости пластины, образующей стенку щелевого канала с пульсирующим слоем вязкой несжимаемой жидкости при заданном гармоническом законе пульсации давления на его торце в плоской постановке. Поставленная краевая задача представляет собой нелинейную связанную систему уравнений Навье-Стокса для слоя вязкой несжимаемой жидкости и уравнения динамики пластины (балки-полоски). В качестве краевых условий выступают условия прилипания жидкости к непроницаемым стенкам канала, условия свободного истечения жидкости на торцах канала и условия шарнирного опирания пластины - стенки канала. Сформирован комплекс безразмерных переменных рассматриваемой задачи и выделены малые параметры задачи. В качестве малых параметров выбраны относительная толщина слоя жидкости и относительная амплитуда прогиба пластины. Рассматривая асимптотические разложения по выделенным малым параметрам задачи, осуществили ее линеаризацию методом возмущений. Решение линеаризованной задачи проведено методом заданных форм для режима установившихся гармонических колебаний. При этом на основании граничных условий для пластины-стенки канала форма ее прогиба задана в виде рядов по тригонометрическим функциям от продольной координаты. Найдены закон прогиба упругой стенки канала и распределения гидродинамических параметров в жидкости. Получены частотозависимые функции распределения амплитуд прогиба и динамического давления вдоль канала и частотозависимые функции распределения фазового сдвига прогиба стенки и давления в канале относительно исходного возмущения на торце. На основе расчетов показано, что резонансные колебания упругой стенки канала, возбуждаемые незначительными пульсациями давления на его торце, могут вызывать существенные изменения динамического давления и являться основной причиной вибрационной кавитации в жидкости.

Ключевые слова: математическое моделирование, плоский канал, гидродинамические параметры, кавитация, упругая стенка, пульсирующий слой, пульсация давления.
\end{abstract}




\author{
R.V. Ageev ${ }^{1}$, E.L. Kuznetsova ${ }^{2}$, N.I. Kulikov², \\ L.I. Mogilevich ${ }^{1}$ V.S. Popov ${ }^{3}$ \\ ${ }^{1}$ Volga Region Branch of Moscow State University of Means \\ of Communication, Saratov, Russian Federation \\ ${ }^{2}$ Moscow Aviation Institute (National Research University), \\ Moscow, Russian Federation \\ ${ }^{3}$ Saratov State Technical University of name Yu.A. Gagarin, \\ Saratov, Russian Federation
}

\title{
MATHEMATICAL MODEL OF MOVEMENT OF A PULSING LAYER OF VISCOUS LIQUID IN THE CHANNEL WITH AN ELASTIC WALL
}

\begin{abstract}
The problem of hydroelasticity of the plate forming at wall of the slot-hole channel with a pulsing layer of viscous incompressible liquid at the set harmonious law of a pulsation of pressure at its end face in flat statement is put and analytically solved. The set regional task represents nonlinear related system of the equations of Navier-Stokes for a layer of viscous incompressible liquid and the equation of dynamics of a plate (beam strip). Conditions of sticking of liquid act as regional conditions to impenetrable walls of the channel, a condition of the free expiration of liquid at end faces of the channel and a condition of a hinged supporting of a plate wall of the channel. The complex of dimensionless variables of a considered task is created and small parameters of a task are allocated. As small parameters we have chosen the relative thickness of a layer of liquid and relative amplitude of a deflection of a plate. Considering asymptotic decomposition in the allocated small parameters of a task we have carried out its linearization by a method of indignations. The solution of the linearized task is obtained by a method of the set forms for a mode of the established harmonic oscillations. Thus, proceeding from boundary conditions for a channel plate wall, the form of its deflection is set in the form of ranks on trigonometrical functions from longitudinal coordinate. The law of a deflection of an elastic wall of the channel and distribution of hydrodynamic parameters are found in liquid. We have obtained frequency dependent functions of distribution of amplitudes of a deflection and dynamic pressure along the channel and frequency dependent functions of distribution of phase shift of a deflection of a wall and pressure in the channel of rather initial indignation at an end face. On the basis of calculations it is shown that resonant fluctuations of an elastic wall of the channel, pressure excited by insignificant pulsations at its end face, can cause essential changes of dynamic pressure and be the main reason of vibration cavitation in liquid.

Keywords: mathematical modelling, flat channel, hydrodynamic parameters, cavitation, elastic wall, a pulsing layer, pressure pulsation.
\end{abstract}

С движением слоя жидкости в плоском канале приходится сталкиваться при исследовании широкого круга проблем, связанных с нормальным функционированием системы охлаждения и подачи топлива в двигателях аэрокосмических систем, а также в энергетике и гидроприводах сложных механических систем, используемых в авиационной и космической отраслях [1-3]. В большинстве случаев ограничиваются рассмотрением стационарного движения жидкости в канале, образованном абсолютно твердыми стенками. В [4, 5] рас- 
смотрены случаи движения слоя вязкой несжимаемой жидкости в канале с абсолютно твердыми стенками в рамках теории гидродинамической смазки. В то же время актуальными являются исследования причин и условий возникновения кавитации в жидкости, находящейся в канале. Например, в работах [6, 7] представлено исследование кавитации в охлаждающей жидкости двигателей внутреннего сгорания. В [6] экспериментально обосновано, что основной причиной вибрационной кавитации является упругая податливость стенок канала, и рассмотрены колебания стенки канала как свободные колебания цилиндрической оболочки без учета ее взаимодействия с жидкостью. В работе [7] исследование причин возникновения кавитации рассмотрено на основе решения задачи взаимодействия упругой балки-полоски с идеальной жидкостью. Однако учет вязкости жидкости видится важным, так как именно ею обусловлены демпфирующие свойства в данной колебательной системе и ограниченность амплитуд колебаний стенки канала на резонансной частоте. В работе [8] представлено исследование гидроупругих колебаний балки в потоке вязкой жидкости применительно к пьезопреобразователям, а в работе [9] проведено исследование плоского двухфазного течения вязкой жидкости в канале переменного сечения с твердыми стенками и учетом кавитационных эффектов.

С другой стороны, в работе [10] рассмотрен вопрос взаимодействия вибрирующих дисков со слоем вязкой несжимаемой жидкости, находящейся между ними, в том числе когда один из них представлен круглой упругой пластиной. Показано, что на резонансных частотах колебаний стенок канала амплитуда давления жидкости может изменяться на несколько порядков и становиться существенно меньше давления насыщенного пара. Аналогичное исследование в плоской постановке для пластин проведено в [11], а для круглых трехслойных пластин в [12]. Вибрация круглой пластины на свободной поверхности идеальной несжимаемой жидкости рассмотрена в [13]. При этом рассматривается область в жидкости, ограниченная жестким дном и цилиндрической поверхностью. Колебания круглой пластины, погруженной в воду со свободной поверхностью, исследованы в [14]. В работе [15] получено и исследовано интегродифференциальное уравнение для малых поперечных колебаний прямолинейного упругого трубопровода с идеальной несжимаемой жидкостью. При этом колебания трубопровода описываются в линейной постановке в балочном приближении. 
В предлагаемой работе для исследования проблем возникновения вибрационной кавитации в жидкости приведено решение задачи о нестационарном движении пульсирующего слоя вязкой жидкости, взаимодействующей с упругой стенкой плоского канала, с учетом особенностей торцевого истечения.

Рассмотрим канал, условно представленный на рис. 1.

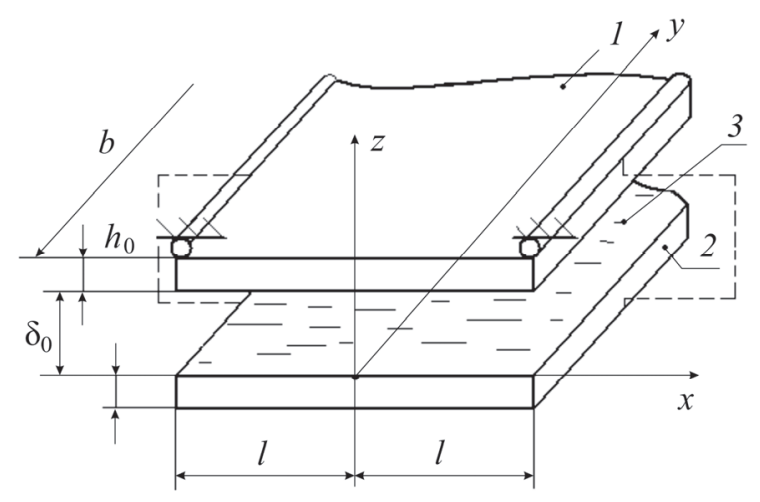

Рис. 1. Схема плоского канала с упругими стенками

Канал образован двумя параллельными непроницаемыми стенками 1, 2 одинаковых геометрических размеров, между которыми движется пульсирующий слой вязкой несжимаемой жидкости за счет заданного закона изменения давления на торцах. Стенка 2 считается абсолютно жесткой, а стенка 1 представляет собой упругую пластину толщиной $h_{0}$ с шарнирным опиранием на торцах. Геометрический размер канала $2 \ell$ значительно меньше его размера $b$, а толщина слоя жидкости (расстояние между стенками) в невозмущенном состоянии $\delta_{0}$ значительно меньше $2 \ell$. Вследствие пульсации давления возникают изгибные колебания стенки 1 , при этом амплитуда ее упругих перемещений значительно меньше $\delta_{0}$.

На торцах канала истечение в полости, заполненные той же жидкостью, можно считать струйным. Для определенности будем далее считать, что в левой полости давление постоянно, $p_{0}$, а в правой полости оно имеет постоянную составляющую и гармонически изменяющуюся по времени составляющую $p_{0}+p_{1}^{+}(\omega t)$. Закон изменения давления на правом торце представим в виде 


$$
p_{1}^{+}=p_{1 m}^{+} f_{p}(\omega t), f_{p}(\omega t)=\exp (i \omega t),
$$

где $p_{1 m}^{+}$- амплитуда пульсаций давления на торцах канала; $\omega$ - частота пульсаций; $f_{p}(\omega t)$ - закон изменения давления.

Введем в рассмотрение декартову систему координат $x, y, z$, связанную с абсолютно твердой стенкой 1 . Учитывая, что $2 \ell<<b$, будем считать канал неограниченным в направлении оси $y$, то есть перейдем к рассмотрению плоской задачи. В этом случае уравнения динамики вязкой несжимаемой жидкости в канале имеют вид

$$
\begin{gathered}
\frac{\partial V_{x}}{\partial t}+V_{x} \frac{\partial V_{x}}{\partial x}+V_{z} \frac{\partial V_{x}}{\partial z}=-\frac{1}{\rho} \frac{\partial p}{\partial x}+v\left(\frac{\partial^{2} V_{x}}{\partial x^{2}}+\frac{\partial^{2} V_{x}}{\partial z^{2}}\right), \\
\frac{\partial V_{z}}{\partial t}+V_{x} \frac{\partial V_{z}}{\partial x}+V_{z} \frac{\partial V_{z}}{\partial z}=-\frac{1}{\rho} \frac{\partial p}{\partial z}+v\left(\frac{\partial^{2} V_{z}}{\partial x^{2}}+\frac{\partial^{2} V_{z}}{\partial z^{2}}\right), \frac{\partial V_{x}}{\partial x}+\frac{\partial V_{z}}{\partial z}=0
\end{gathered}
$$

где $p$ - давление; $\rho, v$ - плотность и кинематический коэффициент вязкости жидкости; $V_{x}, V_{z}$ - проекции скорости движения жидкости на оси координат.

Уравнения динамики жидкости дополняются граничными условиями:

условиями прилипания жидкости к стенкам канала $[10,11,16]$ :

$$
V_{x}=0, V_{z}=0 \text { при } z=0 ; V_{x}=\frac{\partial u}{\partial t}, V_{z}=\frac{\partial w}{\partial t} \text { при } z=\delta_{0}+w
$$

и условиями ее свободного торцевого истечения, заключающимися в совпадении давления жидкости на торце канала с давлением в полости:

$$
p=p_{0}+p_{1}^{+}(\omega t) \text { при } x=\ell ; p=p_{0} \text { при } x=-\ell .
$$

Здесь $w$ - прогиб стенки 1 канала; $u$ - продольное перемещение стенки 1 канала.

Для упругой пластины - стенки канала выполняется условие $2 \ell<<b$, поэтому уравнения ее движения представляют собой уравнения динамики балки-полоски

$$
D \frac{\partial^{4} w}{\partial x^{4}}+\rho_{0} h_{0} \frac{\partial^{2} w}{\partial t^{2}}=-q_{z z}
$$


где $q_{z z}=-p+2 \rho v\left(\partial V_{z} / \partial z\right)$ - нормальное напряжение, действующее со стороны жидкости на пластину; $D=E h_{0}^{3} /\left(12\left(1-\mu_{0}^{2}\right)\right)$ - цилиндрическая жесткость пластины; $h_{0}$ - толщина пластины; $\rho_{0}$ - плотность материала пластины; $\mu_{0}$ - коэффициент Пуассона материала пластины; $E$ - модуль Юнга материала пластины.

Граничные условия уравнений (5) - условия шарнирного опирания на торцах:

$$
w=\partial^{2} w / \partial x^{2}=0 \text { при } x= \pm \ell .
$$

Введем в рассмотрение безразмерные переменные

$$
\begin{gathered}
\psi=\delta_{0} / \ell<<1, \lambda=w_{m} / \delta_{0}<<1, \operatorname{Re}=\delta_{0}^{2} \omega / v, \tau=\omega t, \xi=x / \ell, \zeta=z / \delta_{0}, \\
V_{z}=w_{m} \omega U_{\zeta} ; V_{x}=w_{m} \omega U_{\xi} / \psi ; p=p_{0}+w_{m} \rho v \omega\left(\delta_{0} \psi^{2}\right)^{-1} P ; w=w_{m} W, \\
u=u_{m} U ; p^{+}=w_{m} \rho v \omega\left(\delta_{0} \psi^{2}\right)^{-1} P^{+} .
\end{gathered}
$$

Здесь $w_{m}$ - амплитуда прогиба пластины; $W$ - безразмерный прогиб пластины; $u_{m}$ - амплитуда продольного перемещения пластины; $U$ безразмерное продольное перемещение пластины; $\psi, \lambda, \operatorname{Re}-$ параметры, характеризующие задачу.

Подставляя введенные переменные (7) в задачу (1)-(6), получим задачу гидроупругости плоского канала в безразмерном виде, включающую уравнения динамики вязкой несжимаемой жидкости

$$
\begin{gathered}
\operatorname{Re}\left[\frac{\partial U_{\xi}}{\partial \tau}+\lambda\left(U_{\xi} \frac{\partial U_{\xi}}{\partial \xi}+U_{\zeta} \frac{\partial U_{\xi}}{\partial \zeta}\right)\right]=-\frac{\partial P}{\partial \xi}+\psi^{2} \frac{\partial^{2} U_{\xi}}{\partial \xi^{2}}+\frac{\partial^{2} U_{\xi}}{\partial \zeta^{2}}, \\
\psi^{2} \operatorname{Re}\left[\frac{\partial U_{\zeta}}{\partial \tau}+\lambda\left(U_{\xi} \frac{\partial U_{\zeta}}{\partial \xi}+U_{\zeta} \frac{\partial U_{\zeta}}{\partial \zeta}\right)\right]=-\frac{\partial P}{\partial \zeta}+\psi^{2}\left[\psi^{2} \frac{\partial^{2} U_{\zeta}}{\partial \xi^{2}}+\frac{\partial^{2} U_{\zeta}}{\partial \zeta^{2}}\right], \\
\frac{\partial U_{\xi}}{\partial \xi}+\frac{\partial U_{\zeta}}{\partial \zeta}=0 ;
\end{gathered}
$$

уравнения динамики упругой стенки канала

$$
\frac{D w_{m}}{\ell^{4}} \frac{\partial^{4} W}{\partial \xi^{4}}+\rho_{0} h_{0} \omega^{2} w_{m} \frac{\partial^{2} W}{\partial \tau^{2}}=p_{0}+\frac{w_{m} \rho v \omega}{\delta_{0} \psi^{2}}\left(P-2 \psi^{2} \frac{\partial U_{\zeta}}{\partial \zeta}\right) .
$$


Граничные условия (3), (4), (6) запишутся в виде

$$
\begin{gathered}
U_{\xi}=\psi \frac{u_{m}}{w_{m}} \frac{\partial U}{\partial \tau}, U_{\zeta}=\frac{\partial W}{\partial \tau} \text { при } \zeta=1+\lambda W ; U_{\xi}=0, U_{\zeta}=0 \text { при } \zeta=0, \\
P=P^{+}(\tau) \text { при } \xi=1 ; P=0 \text { при } \xi=-1, \\
W=\partial^{2} W / \partial \xi^{2}=0 \text { при } \xi= \pm 1 .
\end{gathered}
$$

В предлагаемой постановке $\psi$ является безразмерным малым параметром, отношение $u_{m} / w_{m}$ порядка единицы, следовательно, в нулевом приближении по $\psi$ уравнения (8), (9) и граничные условия (10) упрощаются, то есть в них можно положить равными нулю члены порядка $\psi$ и $\psi^{2}$. Далее, рассматривая асимптотические разложения по малому параметру $\lambda \ll<$ [17] $P=P_{0}+\lambda P_{1}+\ldots, U_{\xi}=U_{\xi 0}+\lambda U_{\xi 1}+. ., U_{\zeta}=$ $=U_{\zeta 0}+\lambda U_{\zeta 1}+\ldots$ и ограничиваясь только первым членом разложения, получим линеаризованную задачу гидроупругости плоского канала, включающую в себя:

уравнения динамики слоя жидкости

$$
\operatorname{Re} \frac{\partial U_{\xi 0}}{\partial \tau}=-\frac{\partial P_{0}}{\partial \xi}+\frac{\partial^{2} U_{\xi 0}}{\partial \zeta^{2}}, \frac{\partial P_{0}}{\partial \zeta}=0, \frac{\partial U_{\xi 0}}{\partial \xi}+\frac{\partial U_{\zeta 0}}{\partial \zeta}=0
$$

с граничными условиями

$$
\begin{gathered}
U_{\xi 0}=0, U_{\zeta 0}=\partial W / \partial \tau \text { при } \zeta=1 ; U_{\xi 0}=0, U_{\zeta 0}=0 \text { при } \zeta=0 ; \\
P=P^{+}(\tau) \text { при } \xi=1 ; P=0 \text { при } \xi=-1
\end{gathered}
$$

и уравнения движения пластинки - стенки канала

$$
\frac{D w_{m}}{\ell^{4}} \frac{\partial^{4} W}{\partial \xi^{4}}+\rho_{0} h_{0} \omega^{2} w_{m} \frac{\partial^{2} W}{\partial \tau^{2}}=p_{0}+\frac{w_{m} \rho v \omega}{\delta_{0} \psi^{2}} P
$$

с граничными условиями

$$
W=\partial^{2} W / \partial \xi^{2}=0 \text { при } \xi= \pm 1 .
$$

Решение уравнений (13) с учетом граничных условий (14), (15) и гармонического характера изменения по времени прогиба пластины стенки канала имеет вид 


$$
\begin{gathered}
U_{\xi 0}=\frac{1}{2 \varepsilon^{2}}\left[\frac{\partial^{2} P_{0}}{\partial \xi \partial \tau}+\frac{\partial^{2} P_{0}}{\partial \xi \partial \tau} \bar{\Psi}(\zeta)+\frac{\partial P_{0}}{\partial \xi} \bar{\Phi}(\zeta)\right] \\
U_{\zeta 0}=\frac{1}{2 \varepsilon^{2}}\left[\frac{\partial^{3} P_{0}}{\partial \xi^{2} \partial \tau}(1-\zeta)+\frac{\partial^{3} P_{0}}{\partial \xi^{2} \partial \tau} \bar{\Psi}_{1}(\zeta)+\frac{\partial^{2} P_{0}}{\partial \xi^{2}} \bar{\Phi}_{1}(\zeta)\right]+\frac{\partial W}{\partial \tau}, \\
P_{0}=\int_{-1}^{\xi} \int\left(2 \varepsilon^{2} \alpha \frac{\partial^{2} W}{\partial \tau^{2}}+12 \gamma \frac{\partial W}{\partial \tau}\right) d \xi d \xi-\frac{1}{2}(\xi+1) \int_{-1}^{1} \int\left(2 \varepsilon^{2} \alpha \frac{\partial^{2} W}{\partial \tau^{2}}+12 \gamma \frac{\partial W}{\partial \tau}\right) d \xi d \xi+ \\
+P_{1}^{+} / 2+\xi P_{1}^{+} / 2 .
\end{gathered}
$$

Здесь введены обозначения

$$
\begin{gathered}
\varepsilon(\omega)=\sqrt{\operatorname{Re} / 2}, F_{1}(\varepsilon \zeta)=\operatorname{ch} \varepsilon \zeta \cos \varepsilon \zeta, F_{3}(\varepsilon \zeta)=\frac{1}{2} \operatorname{sh} \varepsilon \zeta \sin \varepsilon \zeta, \\
F_{2}(\varepsilon \zeta)=\frac{1}{2}[\operatorname{ch} \varepsilon \zeta \sin \varepsilon \zeta+\operatorname{sh} \varepsilon \zeta \cos \varepsilon \zeta], F_{4}(\varepsilon \zeta)=\frac{1}{4}[\operatorname{ch} \varepsilon \zeta \sin \varepsilon \zeta-\operatorname{sh} \varepsilon \zeta \cos \varepsilon \zeta], \\
D_{1}=\frac{\operatorname{sh} \varepsilon-\sin \varepsilon}{\cos \varepsilon+\operatorname{ch} \varepsilon}, D_{2}=\frac{\sin \varepsilon+\operatorname{sh} \varepsilon}{\cos \varepsilon+\operatorname{ch} \varepsilon}, \\
\gamma(\omega)=\frac{1}{6} \frac{\varepsilon^{3}(\operatorname{sh} \varepsilon-\sin \varepsilon)}{\varepsilon^{2}(\operatorname{ch} \varepsilon+\cos \varepsilon)-2 \varepsilon(\operatorname{sh} \varepsilon+\sin \varepsilon)+2(\operatorname{ch} \varepsilon-\cos \varepsilon)} \\
\alpha(\omega)=\frac{\varepsilon(\varepsilon(\operatorname{ch} \varepsilon+\cos \varepsilon)-(\operatorname{sh} \varepsilon+\sin \varepsilon))}{\varepsilon^{2}(\operatorname{ch} \varepsilon+\cos \varepsilon)-2 \varepsilon(\operatorname{sh} \varepsilon+\sin \varepsilon)+2(\operatorname{ch} \varepsilon-\cos \varepsilon)} \\
\bar{\Psi}(\zeta)=F_{2}(\varepsilon \zeta) D_{1}-F_{1}(\varepsilon \zeta)-2 F_{4}(\varepsilon \zeta) D_{2}, \\
\bar{\Phi}(\zeta)=2 F_{3}(\varepsilon \zeta)-F_{2}(\varepsilon \zeta) D_{2}-2 F_{4}(\varepsilon \zeta) D_{1}, \bar{\Psi}_{1}(\zeta)=\int_{\zeta}^{1} \bar{\Psi}(\zeta) d \zeta \\
\bar{\Phi}_{1}(\zeta)=\int_{\zeta}^{1} \bar{\Phi}(\zeta) d \zeta .
\end{gathered}
$$

Решение уравнений (16) с учетом краевых условий (17) представим в виде

$$
W=\sum_{k=1}^{\infty}\left(R_{k}(\tau)+R_{k}^{0}\right) \cos ((2 k-1) \pi \xi) / 2+Q_{k}(\tau) \sin k \pi \xi .
$$

Верхний индекс 0 в (19) означает решение, соответствующее постоянному уровню давления $p_{0}$, не зависящему от $\tau$.

Принимая во внимание линейность уравнения (16) и подставляя в него (19), найденное выражение для давления (18), а также раскладывая оставшиеся члены, входящие в его правую часть в ряды по триго- 
нометрическим функциям, получим уравнение для составляющей, не зависящей от времени $R_{k}^{0}$ :

$$
\sum_{k=1}^{\infty}\left(\frac{2 k-1}{2 \ell} \pi\right)^{4} D w_{m} R_{k}^{0} \cos \frac{2 k-1}{2} \pi \xi=\sum_{k=1}^{\infty} \frac{4(-1)^{k+1}}{(2 k-1) \pi} p_{0} \cos \frac{2 k-1}{2} \pi \xi
$$

и уравнение для неизвестных функций времени $R_{k}$ и $Q_{k}$ :

$$
\begin{gathered}
\sum_{k=1}^{\infty} w_{m}\left[D\left(\frac{2 k-1}{2 \ell} \pi\right)^{4} R_{k} \cos \frac{2 k-1}{2} \pi \xi+D\left(\frac{k \pi}{\ell}\right)^{4} Q_{k} \sin k \pi \xi+\right. \\
\left.+\rho_{0} h_{0} \omega^{2}\left(\frac{d^{2} R_{k}}{d \tau^{2}} \cos \frac{2 k-1}{2} \pi \xi+\frac{d^{2} Q_{k}}{d \tau^{2}} \sin k \pi \xi\right)\right]= \\
=\sum_{k=1}^{\infty}\left[\frac{4(-1)^{k+1}}{(2 k-1) \pi} \frac{p_{1}^{+}}{2} \cos \frac{2 k-1}{2} \pi \xi+\frac{2(-1)^{k+1}}{k \pi} \frac{p_{1}^{+}}{2} \sin k \pi \xi\right]- \\
-w_{m} \sum_{k=1}^{\infty}\left[\left(M_{c k} \omega^{2} \frac{d^{2} R_{k}}{d \tau^{2}}+2 K_{c k} \omega \frac{d R_{k}}{d \tau}\right) \cos \frac{2 k-1}{2} \pi \xi-\right. \\
\left.-\left(M_{s k} \omega^{2} \frac{d^{2} Q_{k}}{d \tau^{2}}+2 K_{s k} \omega \frac{d Q_{k}}{d \tau}\right) \sin k \pi \xi\right] .
\end{gathered}
$$

Учитывая, что $d^{2} R_{k} / d \tau^{2}=-R_{k}, d^{2} Q_{k} / d \tau^{2}=-Q_{k}$, вводя обозначения

$$
\begin{aligned}
& M_{c k}=\frac{\rho v}{\delta_{0} \psi^{2}}\left[\frac{2}{(2 k-1) \pi}\right]^{2} \frac{2 \varepsilon^{2} \alpha}{\omega} ; 2 K_{c k}=\frac{12 \gamma \omega}{2 \varepsilon^{2} \alpha} M_{c k} ; \quad M_{s k}=\frac{\rho v}{\delta_{0} \psi^{2}}\left[\frac{1}{k \pi}\right]^{2} \frac{2 \varepsilon^{2} \alpha}{\omega} ; \\
& 2 K_{s k}=\frac{12 \gamma \omega}{2 \varepsilon^{2} \alpha} M_{s k} ; \quad a_{1 c k}=((2 k-1) \pi / 2 \ell)^{4}-\left[\rho_{0} h_{0}+M_{c k}\right] \omega^{2} / D ; \quad a_{2 c k}= \\
& =2 K_{c k} \omega / D ; a_{1 s k}=(k \pi / \ell)^{4}-\left[\rho_{0} h_{0}+M_{s k}\right] \omega^{2} / D ; a_{2 s k}=2 K_{s k} \omega / D
\end{aligned}
$$

и приравнивая коэффициенты при косинусах и синусах, из (20) находим выражение для $R_{k}^{0}$ :

$$
R_{k}^{0}=(2 \ell /((2 k-1) \pi))^{4}\left(4(-1)^{k+1} /((2 k-1) \pi)\right) p_{0}\left(D w_{m}\right)^{-1},
$$

а из (21) - уравнения для определения $R_{k}(\tau)$ и $Q_{k}(\tau)$ :

$$
a_{1 c k} w_{m} R_{k}+a_{2 c k} w_{m} d R_{k} / d \tau=2(-1)^{k+1} /((2 k-1) \pi D) p_{m}^{+} f_{p}(\tau)
$$




$$
a_{1 s k} w_{m} Q_{k}+a_{2 s k} w_{m} d Q_{k} / d \tau=(-1)^{k+1} /(k \pi D) p_{m}^{+} f_{p}(\tau)
$$

Частные решения уравнений (23), (24), соответствующие гармоническому закону пульсаций давления на торце канала (1), имеют вид

$$
\begin{gathered}
R_{k}=\frac{2(-1)^{k+1}}{(2 k-1) \pi} \frac{p_{m}^{+}}{w_{m} D}\left[A_{c k} \frac{d f_{p}}{d \tau}+B_{c k} f_{p}\right] \\
Q_{k}=\frac{(-1)^{k+1}}{k \pi} \frac{p_{m}^{+}}{w_{m} D}\left[A_{s k} \frac{d f_{p}}{d \tau}+B_{s k} f_{p}\right]
\end{gathered}
$$

где $A_{c k}=-\frac{a_{2 c k}}{a_{1 c k}^{2}+a_{2 c k}^{2}} ; B_{c k}=\frac{a_{1 c k}}{a_{1 c k}^{2}+a_{2 c k}^{2}}, A_{s k}=-\frac{a_{2 s k}}{a_{1 s k}^{2}+a_{2 s k}^{2}}, B_{s k}=\frac{a_{1 s k}}{a_{1 s k}^{2}+a_{2 s k}^{2}}$.

С учетом (25) прогиб стенки 1 имеет вид

$$
\begin{gathered}
w=w_{m} \sum_{k=1}^{\infty}\left\langle\frac{2(-1)^{k+1}}{(2 k-1) \pi}\left\{\left(\frac{2 \ell}{(2 k-1) \pi}\right)^{4} \frac{2 p_{0}}{D w_{m}}+\frac{p_{m}^{+}}{D w_{m}}\left[A_{c k} \frac{d f_{p}}{d \tau}+B_{c k} f_{p}\right]\right\} \times\right. \\
\left.\times \cos \frac{2 k-1}{2} \pi \xi+\frac{(-1)^{k+1}}{k \pi} \frac{p_{m}^{+}}{w_{m} D}\left[A_{s k} \frac{d f_{p}}{d \tau}+B_{s k} f_{p}\right] \sin k \pi \xi\right\rangle= \\
=\frac{p_{0}}{D}\left[\sum_{k=1}^{\infty} \frac{4(-1)^{k+1}}{(2 k-1) \pi}\left(\frac{2 \ell}{(2 k-1) \pi}\right)^{4} \cos \frac{2 k-1}{2} \pi \xi\right]+ \\
+p_{m}^{+} A(\xi, \omega) \exp [i(\tau+\varphi(\xi, \omega))],
\end{gathered}
$$

где $A(\xi, \omega)=\sqrt{C(\xi, \omega)^{2}+B(\xi, \omega)^{2}} ; \varphi(\xi, \omega)=\operatorname{arctg}(C(\xi, \omega) / B(\xi, \omega))$,

$$
\begin{aligned}
& C(\xi, \omega)=\left[\sum_{k=1}^{\infty} \frac{2(-1)^{k+1}}{(2 k-1) \pi} \frac{A_{c k}}{D} \cos \frac{2 k-1}{2} \pi \xi+\sum_{k=1}^{\infty} \frac{(-1)^{k+1}}{k \pi} \frac{A_{s k}}{D} \sin k \pi \xi\right], \\
& B(\xi, \omega)=\left[\sum_{k=1}^{\infty} \frac{2(-1)^{k+1}}{(2 k-1) \pi} \frac{B_{c k}}{D} \cos \frac{2 k-1}{2} \pi \xi+\sum_{k=1}^{\infty} \frac{(-1)^{k+1}}{k \pi} \frac{B_{s k}}{D} \sin k \pi \xi\right] .
\end{aligned}
$$

Используя найденное выражение для прогиба (26), окончательно определяем закон изменения динамического давления в безразмерном виде: 


$$
\begin{gathered}
P_{0}=P_{1}^{+}(\tau) / 2+\xi P_{1}^{+}(\tau) / 2+\frac{p_{m}^{+}}{D w_{m}}\left\langle\sum_{k=1}^{\infty}\left(\frac{2}{(2 k-1) \pi}\right)^{3}(-1)^{k+1} \times\right. \\
\times\left[12 \gamma\left(B_{c k} \frac{d f_{p}}{d \tau}-A_{c k} f_{p}\right)-2 \varepsilon^{2} \alpha\left(A_{c k} \frac{d f_{p}}{d \tau}+B_{c k} f_{p}\right)\right] \cos \left(\frac{2 k-1}{2} \pi \xi\right)+ \\
\left.+\sum_{k=1}^{\infty}\left(\frac{1}{\pi k}\right)^{3}(-1)^{k+1}\left[12 \gamma\left(B_{s k} \frac{d f_{p}}{d \tau}-A_{s k} f_{p}\right)-2 \varepsilon^{2} \alpha\left(A_{s k} \frac{d f_{p}}{d \tau}+B_{s k} f_{p}\right)\right] \sin k \pi \xi\right\rangle
\end{gathered}
$$

и в размерном виде:

$$
p=p_{1}^{+}(\tau)(1+\xi) / 2+p_{m}^{+} \Pi(\xi, \omega) \sin \left(\tau+\varphi_{p}(\xi, \omega)\right)
$$

где $\Pi(\xi, \omega)=\frac{\rho v \omega}{D \delta_{0} \psi^{2}}\left(S(\xi, \omega)^{2}+Q(\xi, \omega)^{2}\right)^{1 / 2}, \varphi(\xi, \omega)=\operatorname{arctg} \frac{S(\xi, \omega)}{Q(\xi, \omega)}$,

$$
\begin{aligned}
S(\xi, \omega)= & \sum_{k=1}^{\infty}\left(\frac{2}{(2 k-1) \pi}\right)^{3}(-1)^{k+1}\left(12 \gamma B_{c k}-2 \varepsilon^{2} \alpha A_{c k}\right) \cos \left(\frac{2 k-1}{2} \pi \xi\right)+ \\
& +\sum_{k=1}^{\infty}\left(\frac{1}{\pi k}\right)^{3}(-1)^{k+1}\left(12 \gamma B_{s k}-2 \varepsilon^{2} \alpha A_{s k}\right) \sin k \pi \xi, \\
Q(\xi, \omega)= & \sum_{k=1}^{\infty}\left(\frac{2}{(2 k-1) \pi}\right)^{3}(-1)^{k}\left(12 \gamma A_{c k}+2 \varepsilon^{2} \alpha B_{c k}\right) \cos \left(\frac{2 k-1}{2} \pi \xi\right)+ \\
& +\sum_{k=1}^{\infty}\left(\frac{1}{\pi k}\right)^{3}(-1)^{k}\left(12 \gamma A_{s k}+2 \varepsilon^{2} \alpha B_{s k}\right) \sin k \pi \xi .
\end{aligned}
$$

Первое слагаемое в выражении прогиба (26) представляет собой прогиб под действием статического давления, а второе слагаемое прогиб, обусловленный динамическим давлением в канале. В выражении для динамического давления (28) слагаемое $p_{1}^{+}(\tau)(1+\xi) / 2$ отражает линейное падение давления вдоль канала, а второе слагаемое представляет собой давление в жидкости, обусловленное ее сдавливанием упругой стенкой канала. При этом хорошо видно, что первое слагаемое динамического давления вдоль канала не превосходит заданного давления на торце $p_{1}^{+}(\tau)$. 
Заметим, что в выражениях (26), (28) получены функции $A(\xi, \omega)$, $\Pi(\xi, \omega)$, которые можно рассматривать как частотозависимые функции распределения относительных амплитуд прогиба и динамического давления вдоль канала. Аналогично функции $\varphi(\xi, \omega), \varphi_{p}(\xi, \omega)$ можно рассматривать как частотозависимые функции распределения фазового сдвига прогиба стенки и давления в канале относительно исходного возмущения на торце. В случае фиксированного значения продольной координаты $\xi$ указанные функции представляют собой амплитудные частотные характеристики и фазовые частотные характеристики прогиба стенки и давления в заданном сечении канала. Таким образом, на базе расчетов функций $A(\xi, \omega), \varphi(\xi, \omega)$ можно исследовать упругие колебания пластины - стенки канала, возбуждаемые пульсацией давления на торце (1) с амплитудой $p_{\mathrm{m}}^{+}$и частотой $\omega$, а при помощи функций П $(\xi, \omega), \varphi_{p}(\xi, \omega)$ исследовать изменение давления вдоль канала, вызванное сдавливанием жидкости упругой пластиной - стенкой канала при ее изгибных колебаниях.

В качестве примера рассмотрим исследование упругих прогибов пластины - стенки канала и динамического давления, обусловленного сдавливанием жидкости упругой стенкой, в центре $(\xi=0)$ канала с параметрами: $\ell=0,1 \mathrm{~m} ; \quad \delta_{0} / \ell=1 / 15 ; \quad b / \ell=5$; $\rho=1,84 \cdot 10^{3} \mathrm{\kappa} \Gamma / \mathrm{m}^{3} ; \quad v=2,5 \cdot 10^{-4} \mathrm{~m}^{2} / \mathrm{c} ; \quad p_{\mathrm{m}}^{+}=1 \Pi \mathrm{a}, \quad \rho_{0}=7,87 \cdot 10^{3} \mathrm{\kappa} \Gamma / \mathrm{m}^{3}$; $h_{0}=3,2 \mathrm{мм} ; E=1,96 \cdot 10^{11} \Pi$ Па $\mu_{0}=0,3$.

Результаты расчетов относительных амплитуд прогибов стенки канала $A(0, \omega)$ и фазового сдвига $\varphi(0, \omega)$ в зависимости от частоты возмущения на торце при удержании одного, двух, трех членов ряда приведены на рис. 2 (кривая 1 - в расчетах удержан один член ряда; кривая 2 - в расчетах удержаны два члена ряда; кривая 3 - в расчетах удержаны три члена ряда). Аналогичные результаты расчетов для относительного давления П $(0, \omega)$ и фазового сдвига $\varphi_{p}(0, \omega)$ приведены на рис. 3. 


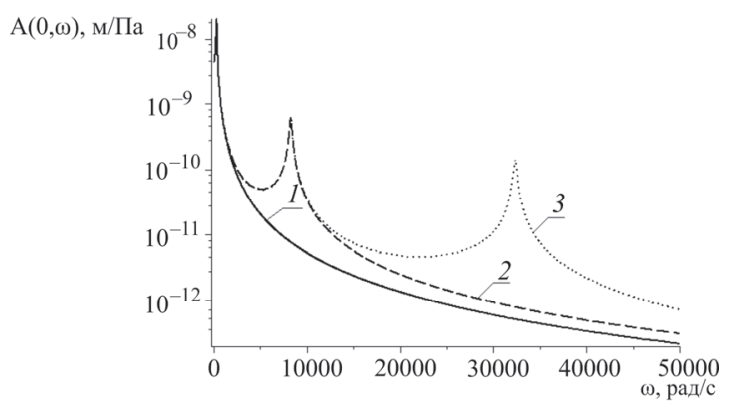

$a$

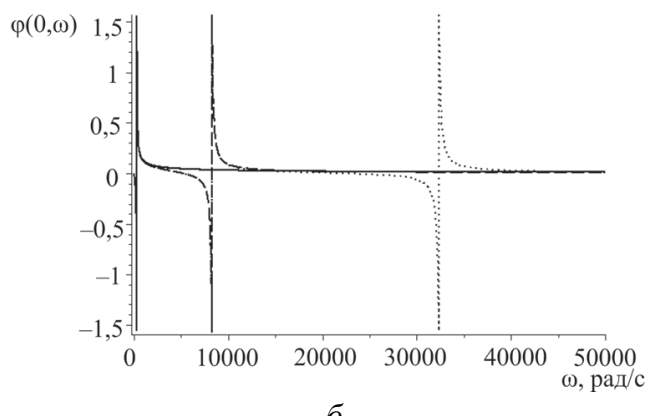

6

Рис. 2. Результаты расчетов относительных амплитуд прогибов $A(0, \omega)$ пластины $(a)$ и фазового сдвига $\varphi(0, \omega)(б)$ в зависимости частоты возмущения

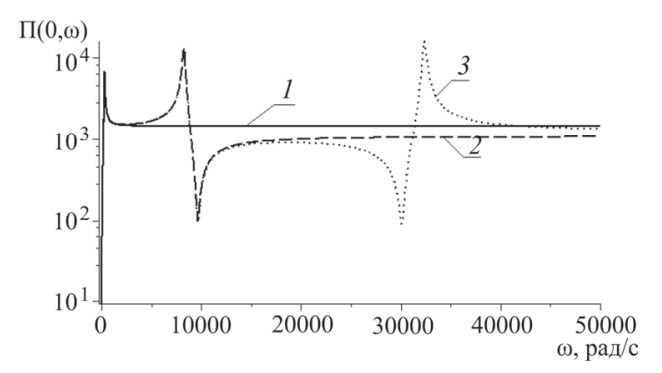

$a$

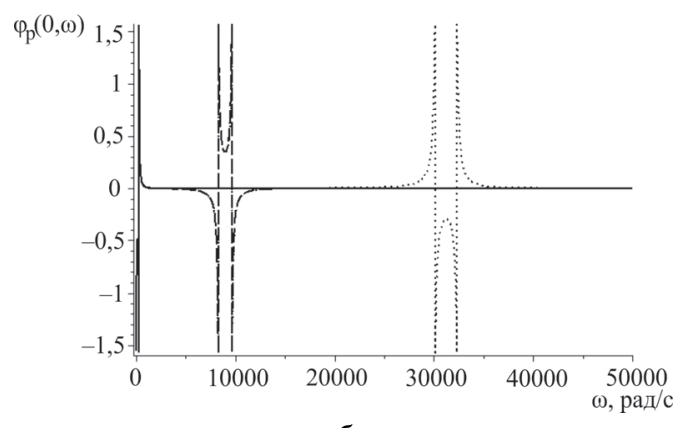

6

Рис. 3. Результаты расчетов относительных амплитуд давления П $(0, \omega)$ жидкости $(a)$ и фазового сдвига $\varphi_{p}(0, \omega)(б)$ в зависимости частоты возмущения 
Проведенное исследование показывает, что наблюдаются пики амплитуд прогибов пластины на резонансных частотах колебаний, данным частотам соответствуют пики давления в жидкости. При этом давление, обусловленное сдавливанием жидкости упругой стенкой, на резонансных частотах превышает заданное давление на торце на 3-4 порядка. Резонансным частотам соответствует смена фазового сдвига с $-\pi / 2$ на $\pi / 2$, на остальных частотах он стремится к нулю. Учет второго и последующих членов ряда в выражении (26) приводит к появлению дополнительных пиков прогибов, а следовательно, и давления, которые соответствуют новым резонансным частотам, расположенным в более высоком частотном диапазоне, чем предыдущие резонансные частоты. Для практических целей достаточно удерживать первые 3-4 члена ряда, так как частоты колебаний стенок канала и пульсации давления на практике не превышают звуковой диапазон. Принимая во внимание гармонический характер изменения давления по времени, можно говорить о расчетах, показывающих, что на резонансных частотах давление может становиться меньше давления насыщенного пара. Таким образом, даже незначительная пульсация давления на торце вызывает значительные изменения давления в канале, обусловленные сдавливанием жидкости упругой стенкой канала на резонансных частотах колебания, что приводит к возникновению вибрационной кавитации в жидкости. Следовательно, гидроупругие колебания стенки канала являются основной причиной резкого роста динамического давления в жидкости на резонансных частотах колебаний. Полученные в работе выражения для прогиба упругой стенки канала и гидродинамических параметров слоя вязкой жидкости могут быть использованы в практике для определения резонансных частот колебаний, соответствующих условиям возникновения кавитации, а также для оценки возможности возникновения резонансных колебаний исходя из заданного частотного диапазона возможных пульсаций давления на торце канала.

Работа выполнена при финансовой поддержке Министерства образования и науки Российской Федерации (проект № 32).

\section{Библиографический список}

1. Башта Т.М. Машиностроительная гидравлика. - М.: Машиностроение, 1971. -672 с.

2. Идельчик И.Е. Аэродинамика промышленных аппаратов. - М.; Л.: Энергия, 1964. - 289 с. 
3. Александров В.Ю., Климовский К.К., Маслов Д.А. Движение жидкости в канале с изменением ее массы // Изв. РАН. Энергетика. 2011. - № 1. - С. 88-94.

4. Лойцянский Л.Г. Механика жидкости и газа. - М.: Дрофа, 2003. $840 \mathrm{c}$.

5. Слезкин Н.А. Динамика вязкой несжимаемой жидкости. - М.: Гостехиздат, 1955. - 520 с.

6. Иванченко Н.Н., Скурдин А.А., Никитин М.Д. Кавитационные разрушения дизелей. - Л.: Машиностроение, 1970. - 152 с.

7. Индейцев Д.А., Полипанов И.С., Соколов С.К. Расчет кавитационного ресурса втулки судовых двигателей // Проблемы машиностроения и надежности машин. - 1994. - № 4. - С. 59-64.

8. Akcabay D.T., Young Y.L. Hydroelastic response and energy harvesting potential of flexible piezoelectric beams in viscous flow // Physics of Fluids. - 2012. - Vol. 24. - Iss. 5.

9. Маркина Н.Л., Ревизников Д.Л., Черкасов С.Г. Исследование кавитационных процессов в канале переменного сечения // Изв. РАН. Энергетика. - 2012. - № 1. - С. 109-118.

10. Mogilevich L.I., Popov V.S. Investigation of the interaction between a viscous incompressible fluid layer and walls of a channel formed by coaxial vibrating discs // Fluid Dynamics. - 2011. - Vol. 46. - No. 3. - P. 375-388.

11. Mogilevich L.I., Popov V.S., Popova A.A., Dynamics of interaction of elastic elements of a vibrating machine with the compressed liquid layer lying between them // Journal of Machinery Manufacture and Reliability. - 2010. - Vol. 39. - No. 4. - P. 322-331.

12. Ageev R.V., Mogilevich L.I., Popov V.S. Vibrations of the Walls of a Slot Channel with a Viscous Fluid Formed by Three_Layer and Solid Disks // Journal of Machinery Manufacture and Reliability. - 2014. - Vol. 43. No. 1. - P. 1-8.

13. Amabili M. Vibrations of circular plates resting on a sloshing liquid: Solution of the fully coupled problem // Journal of Sound and Vibration. - 2001. - Vol. 245. - Iss. 2. - P. 261-283.

14. Askari E., Jeong K.-H., Amabili M. Hydroelastic vibration of circular plates immersed in a liquid-filled container with free surface // Journal of Sound and Vibration. - 2013. - Vol. 332. - Iss. 12. - P. 3064-3085.

15. Veklich N.A. Equation of Small Transverse Vibrations of an Elastic Pipeline Filled with a Transported Fluid // Mechanics of Solids. - 2013. Vol. 48. - No. 6. - P. 673-681. 
16. Аэрогидроупругость конструкций / А.Г. Горшков, В.И. Морозов, А.Т. Пономарев, Ф.Н. Шклярчук. - М.: Физматлит, 2000.

17. Van Dyke, M. Perturbation methods in fluid mechanics // The Parabolic Press, Stanford, Calif., 1975.

\section{References}

1. Bashta T.M. Mashinostroitelnaia gidravlika [Engineering Hydraulics]. Moscow: Mashinostroenie, 1971. 672 p.

2. Idelchik I.E. Aerodinamika promyshlennykh apparatov [Aerodynamics of industrial devices]. Moscow; Leningrad: Energiya, 1964. 289 p.

3. Alexandrov V.U., Klimovsky K.K., Maslov D.A. Dvizhenie zhidkosti $\mathrm{v}$ kanale $\mathrm{s}$ izmeneniem ee massy [Liquid Fluid motion in channel, while mass of fluid is changed]. Izvestiya Rossiiskoi Akademii Nauk. Energetika, 2011, no. 1, pp. 88-94.

4. Loitsyanskii L.G., Mekhanika zhidkosti i gaza [Mechanics of Liquid and Gas]. Moscow: Drofa, 2003. 840 p.

5. Slezkin N.A. Dinamika vyazkoi neszhimaemoi zhidkosti [Dynamics of a Viscous Incompressible Fluid]. Moscow: Gostekhizdat, $1955.520 \mathrm{p}$.

6. Ivanchenko N.N., Skuridin A.A., Nikitin, M.D. Kavitatsionnoe razrushenie dizelei [Cavitation Destruction in Diesel Motors]. Leningrad: Mashinostroenie, 1970. 152 p.

7. Indeitsev D.A., Polypanov I.S., Sokolov S.K. Raschet kavitatsionnogo resursa vtulki sudovykh dvigatelei [Calculation of Cavitation LifeTime of Ship Engine Liner]. Problemy Mashinostraeniya i Nadezhnos'ti Mashin., 1994, no. 4. pp. 59-64.

8. Akcabay, D.T., Young, Y.L. Hydroelastic response and energy harvesting potential of flexible piezoelectric beams in viscous flow. Physics of Fluids, 2012, vol. 24, iss. 5.

9. Markina N.L., Reviznikov D.L., Cherkasov S.G. Research of cavitational processes in the channel of variable section. Izv. Russian Academy of Sciences. Power, 2012, no. 1, pp. 109-118.

10. Mogilevich L.I., Popov V.S. Investigation of the interaction between a viscous incompressible fluid layer and walls of a channel formed by coaxial vibrating discs. Fluid Dynamics, 2011, vol. 46, no. 3, pp. 375-388.

11. Mogilevich L.I., Popov V.S., Popova A.A., Dynamics of interaction of elastic elements of a vibrating machine with the compressed liquid 
layer lying between them. Journal of Machinery Manufacture and Reliability, 2010, vol. 39, no. 4, pp. 322-331.

12. Ageev R.V., Mogilevich L.I., Popov V.S. Vibrations of the Walls of a Slot Channel with a Viscous Fluid Formed by Three_Layer and Solid Disks. Journal of Machinery Manufacture and Reliability, 2014, vol. 43, no. 1, pp. 1-8.

13. Amabili M. Vibrations of circular plates resting on a sloshing liquid: Solution of the fully coupled problem. Journal of Sound and Vibration, 2001, vol. 245 , iss. 2 , pp. 261-283

14. Askari E., Jeong K.-H., Amabili M. Hydroelastic vibration of circular plates immersed in a liquid-filled container with free surface. Journal of Sound and Vibration, 2013, vol. 332, iss. 12, pp. 3064-3085

15. Veklich N.A. Equation of Small Transverse Vibrations of an Elastic Pipeline Filled with a Transported Fluid. Mechanics of Solids, 2013, vol. 48, no. 6, pp. 673-681

16. Gorshkov A.G., Morozov V.I. Ponomarev A.T. Shklyarchuk F.N. Aerouprugost konstruktsii [Aerogidroelasticity of designs]. Moscow: Fizmathlit, 2000.

17. Van Dyke M. Perturbation methods in fluid mechanics. The Parabolic Press. Stanford, Calif., 1975.

\section{Об авторах}

Агеев Ростислав Васильевич (Саратов, Россия) - кандидат технических наук, доцент Поволжского филиала Московского государственного университета путей сообщения $(410790$, г. Саратов, ул. Астраханская, 1a, e-mail: arvbs@mail.ru).

Кузнецова Екатерина Львовна (Москва, Россия) - доктор физико-математических наук, профессор, ведущий научный сотрудник НИО-9 МАИ (125993, г. Москва, А-80, ГСП-3, Волоколамское шоссе, д.4, lareyna@mail.ru).

Куликов Николай Иванович (Москва, Россия) - доктор биологических наук, кандидат технических наук, профессор, профессор кафедры электроэнергетических, электромеханических и биотехнических систем Московского авиационного института (национального исследовательского университета (125993, г. Москва, А-80, ГСП-3, Волоколамское шоссе, д. 4, e-mail: nik@mai.ru). 
Могилевич Лев Ильич (Саратов, Россия) - доктор технических наук, профессор, профессор Поволжского филиала Московского государственного университета путей сообщения (410790, г. Саратов, ул. Астраханская, 1a, e-mail: mogilevich@sgu.ru).

Попов Виктор Сергеевич (Саратов, Россия) - доктор технических наук, профессор, профессор Саратовского государственного технического университета имени Ю.А. Гагарина (410054, г. Саратов, ул. Политехническая, 77, e-mail: vic_p@bk.ru).

\section{About the authors}

Rostislav V. Ageev (Saratov, Russian Federation) - Ph.D. in Technical Sciences, Associate Professor of Volga Region Branch of Moscow State University of Means of Communication (1a, Astrakhanskaya str., 410790, Saratov, Russian Federation, e-mail: arvbs@mail.ru).

Ekaterina L. Kuznetsova (Moscow, Russian Federation) - Doctor of Physical and Mathematical Sciences, Professor, Leading Researcher of NIO-9 of Moscow Aviation Institute (National Research University) (4, Volokolamskoye shosse, 125993, A-80, GSP-3, Moscow, Russian Federation, e-mail: lareyna@mail.ru).

Nikolay I. Kulikov (Moscow, Russian Federation) - Doctor of Physical and Mathematical Sciences, Ph.D. in Technical Sciences, Professor, Professor of Electrical Power, Electromechanical and Biotechnical Systems Department of Moscow Aviation Institute (National Research University) (4, Volokolamskoye shosse, 125993, A-80, GSP-3, Moscow, Russian Federation, e-mail: nik@mai.ru).

Lev I. Mogilevich (Saratov, Russian Federation) - Doctor of Technical Sciences, Professor of Volga Region Branch of Moscow State University of Railway Engineering (1a, Astrakhanskaya str., 410790, Saratov, Russian Federation, e-mail: mogilevich@sgu.ru).

Victor S. Popov (Saratov, Russian Federation) - Doctor of Technical Sciences, Professor of Saratov State Technical University of name Yu.A. Gagarin (77, Politekhnicheskaya str., 410054, Saratov, Russian Federation, e-mail: vic_p@bk.ru).

Получено 11.07.2014 
Просьба ссылаться на эту статью в русскоязычных источниках следующим образом:

Математическая модель движения пульсирующего слоя вязкой жидкости в канале с упругой стенкой / Р.В. Агеев, Е.Л. Кузнецова, Н.И. Куликов, Л.И. Могилевич, В.С. Попов // Вестник Пермского национального исследовательского политехнического университета. Механика. - 2014. - № 3. - С. 17-35. DOI: 10.15593/perm.mech/2014.3.02

Please cite this article in English as:

Ageev R.V., Kuznetsova E.L., Kulikov N.I., Mogilevich L.I., Popov V.S. Mathematical model of movement of a pulsing layer of viscous liquid in the channel with an elastic wall. PNRPU Mechanics Bulletin. 2014. No. 3. Pp. 17-35. DOI: 10.15593/perm.mech/2014.3.02 